PROCEEDINGS OF THE AMERICAN MATHEMATICAL SOCIETY

Volume 124, Number 2, February 1996

\title{
WHALES AND THE UNIVERSAL COMPLETION
}

\author{
GERARD BUSKES AND ARNOUD VAN ROOIJ \\ (Communicated by Palle E. T. Jorgensen) \\ Dedicated to the memory of Bill Causey
}

\begin{abstract}
In this paper we introduce whales in the collection of subsets of the Boolean algebra of bands in a Dedekind complete Riesz space and employ them to give a short (and constructive) proof of the existence of universal completions for Archimedean Riesz spaces.
\end{abstract}

The existence of a Dedekind completion for an Archimedean Riesz space, emulating the construction of the real numbers from the rationals, in essence goes back to Dedekind. The construction, using Dedekind cuts, is valid in Zermelo-Fraenkel set theory.

A Riesz space is called laterally complete if each of its disjoint subsets has a least upper bound. The existence of a lateral completion for Archimedean Riesz spaces can be traced to Maeda and Ogasawara. A laterally complete space which at the same time is Dedekind complete is called universally complete. Fremlin in [9], for good reason naming universally complete spaces inextensible, suggested that the existence of a universal completion could be proved without resource to the MaedaOgasawara Representation Theorem. Zaanen, differently than expected from Fremlin's paper, followed suit in [12]. Zaanen does not use the Maeda-Ogasawara Representation Theorem, but he does use the Axiom of Choice. In fact, in spite of the elegance of Zaanen's approach, it falls outside the scope of Zermelo-Fraenkel set theory on many counts and uses the Axiom of Choice repeatedly. We tried to trail Fremlin's hint more closely and use his dominable subsets, but it was not clear to us how to avoid the Axiom of Choice that way either.

In this note we give a different proof which doesn't use representation theorems and is valid in Zermelo-Fraenkel set theory. Moreover, our proof is easier and uses the novel notion of whales, which might be useful in other circumstances. We add to this that the new aspects of our construction (an easy proof instead of a difficult one and the fact that it is AC-free) gain some significance by the fact that the topic of universal completions has gathered momentum by various recent uses in the theory of positive operators, which on its turn shows promise in applications as diverse as economic theories, dynamical systems and partial differential equations.

By the first remark of our paper, we can restrict ourselves without loss of generality to Dedekind complete Riesz spaces. In the following $E$ is a Dedekind complete Riesz space. For unexplained terminology we refer to [1], [2], [3] or [10].

Received by the editors January 10, 1994 and, in revised form, April 4, 1994.

1991 Mathematics Subject Classification. Primary 46A40.

Both authors acknowledge partial support from NATO grant 940605. 


\section{Whales}

Let $\mathcal{B}$ be the Boolean algebra of all bands of $E$. For $e \in E$ and $B \in \mathcal{B}$ we denote the component of $e$ in $B$ by $e_{B}$.

A subset $\mathcal{A}$ of $\mathcal{B}$ is called a whale if

(1) if $A \in \mathcal{A}, B \in \mathcal{B}$ and $B \subset A$, then $B \in \mathcal{A}$ and

(2) $\sup \mathcal{A}=\mathbf{1}(=E)$.

The following easy lemma will often be used implicitly.

Lemma 1. Every union of whales is a whale and if $\mathcal{A}$ and $\mathcal{A}^{\prime}$ are whales, then so is $\mathcal{A} \cap \mathcal{A}^{\prime}\left(=\left\{A \cap A^{\prime}: A \in \mathcal{A}, A^{\prime} \in \mathcal{A}^{\prime}\right\}\right)$.

For a whale $\mathcal{A}$ we define

$$
\begin{aligned}
E_{\mathcal{A}} & =\left\{x \in \prod_{A \in \mathcal{A}} A: x(A)=x(B)_{A} \text { if } A, B \in \mathcal{A} \text { and } A \subset B\right\} \\
& =\left\{x \in \prod_{A \in \mathcal{A}} A: x(A)_{A \cap B}=x(B)_{A \cap B} \text { if } A, B \in \mathcal{A}\right\} .
\end{aligned}
$$

For $x \in E_{\mathcal{A}}$ we call $\mathcal{A}$ the domain of $x$ and write $\mathcal{A}=\operatorname{Dom}_{x}$. The above definition can then be rephrased as

$$
E_{\mathcal{A}}=\left\{x \in \prod_{A \in \mathcal{A}} A: x(A)_{B}=x(A \cap B) \text { if } A \in \operatorname{Dom}_{x}, B \in \mathcal{B}\right\} .
$$

Define a binary relation $\sim$ in $\bigcup_{\mathcal{A}} E_{\mathcal{A}}$ as follows:

$$
x \sim y \Leftrightarrow x=y \text { on } \operatorname{Dom}_{x} \cap \operatorname{Dom}_{y} .
$$

We gather some immediate observations.

Lemma 2. (1) is an equivalence relation.

(2) If $x \sim y$ and $\operatorname{Dom}_{x}=\operatorname{Dom}_{y}$, then $x=y$.

(3) If $x \sim y$ and $\operatorname{Dom}_{x} \subset \operatorname{Dom}_{y}$, then $x$ is the restriction of $y$ to $\operatorname{Dom}_{x}$.

Proof. (2) and (3) are immediate. For the transitivity in (1) we remark the following. If $x \sim y$ and $y \sim z$ and $A \in \operatorname{Dom}_{x} \cap \operatorname{Dom}_{z}$, then

$$
x(A)_{B}=x(A \cap B)=y(A \cap B)=z(A \cap B)=z(A)_{B} \quad \text { for all } B \in \operatorname{Dom}_{y} .
$$

Thus $x(A)=z(A)$ because $\sup \left(\operatorname{Dom}_{y}\right)=\mathbf{1}$.

We also note the following facts.

Lemma 3. (1) Every $E_{\mathcal{A}}$ is a Dedekind complete Riesz space.

(2) If $\mathcal{A}_{1}$ and $\mathcal{A}_{2}$ are whales and $\mathcal{A}_{1} \subset \mathcal{A}_{2}$, then the restriction map $E_{\mathcal{A}_{2}} \rightarrow E_{\mathcal{A}_{1}}$ is a Riesz isomorphism of $E_{\mathcal{A}_{2}}$ onto a Riesz ideal of $E_{\mathcal{A}_{1}}$.

(3) The formula

$$
e \mapsto\left(e_{B}\right)_{B \in \mathcal{B}} \quad(e \in E)
$$

defines a Riesz isomorphism of $E$ onto $E_{\mathcal{B}}$.

Proof. (1) is left to the reader and so is the fact that the range of the map in (2) is an ideal. In order to prove the other part of (2), let $x, y \in E_{\mathcal{A}_{2}}$ and denote the 
restriction map by $\alpha$. Suppose $\alpha(x)=\alpha(y)$ and let $A \in \mathcal{A}_{2}$. Then for all $B \in \mathcal{A}_{1}$ we have

$$
x(A)_{B}=x(A \cap B)=[\alpha(x)](A \cap B)=[\alpha(y)](A \cap B)=y(A \cap B)=y(A)_{B}
$$

and thus $x(A)=y(A)$ because $\sup \left\{B: B \in \mathcal{A}_{1}\right\}=\mathbf{1}$. This proves the injectivity of the restriction map. Also, if $\alpha(x)=z \in E_{\mathcal{A}_{1}}^{+}$for $x \in E_{\mathcal{A}_{2}}$ and $A \in \mathcal{A}_{2}$, then

$$
x(A)_{B}=z(A \cap B)
$$

for all $B \in \mathcal{A}_{1}$, which shows that $\alpha$ is bipositive and hence an isomorphism by Theorem 7.3 in [2].

The injectivity in (3) is immediate. For the surjectivity and the bipositivity let $x \in E_{\mathcal{B}}^{+}$. Then

$$
x(E) \in E^{+}
$$

is the element that is mapped onto $x$ by the formula in (3).

\section{The Universal COMPLETION}

With the notation of the first section we define

$$
\Omega=\bigcup_{\mathcal{A}} E_{\mathcal{A}} / \sim
$$

We want to make $\Omega$ into a Riesz space, in fact into a universally complete Riesz space. With the aid of the Axiom of Choice that can be done easily. Indeed, if at this point one uses the Axiom of Choice, then a choice function $\Omega \rightarrow \bigcup_{\mathcal{A}} E_{\mathcal{A}}$ becomes available. We avoid the AC by virtue of the following lemma.

Lemma 4. Every equivalence class in $\Omega$ contains a unique element with largest domain.

Proof. Let $\xi \in \Omega$. The set $\mathcal{A}_{0}:=\bigcup_{x \in \xi} \operatorname{Dom}_{x}$ is a whale by Lemma 1. Define $x_{0} \in E_{\mathcal{A}_{0}}$ by $x_{0}(A)=x(A)$ if $A \in \operatorname{Dom}_{x}$ for $x \in \xi$. The unique element of the lemma is this $x_{0}$.

For $x \in \bigcup_{\mathcal{A}} E_{\mathcal{A}}$ we denote by $\bar{x}$ the element of the equivalence class of $x$ with largest domain. From now on we will identify $\Omega$ and $\left\{\bar{x}: x \in \bigcup_{\mathcal{A}} E_{\mathcal{A}}\right\}$. For every whale $\mathcal{A}$ put $\Omega_{\mathcal{A}}=\left\{\bar{x}: x \in E_{\mathcal{A}}\right\}$. Trivially, $E_{\mathcal{B}}=\Omega_{\mathcal{B}}$.

If $\mathcal{A}$ is a whale and $x \in E_{\mathcal{A}}$, then $x$ is the restriction of $\bar{x}$ to $\mathcal{A}$. Thus, $x \mapsto \bar{x}(x \in$ $E_{\mathcal{A}}$ ) is a bijection of $E_{\mathcal{A}}$ onto $\Omega_{\mathcal{A}}$, inducing on $\Omega_{\mathcal{A}}$ the structure of a Dedekind complete Riesz space. If $\mathcal{A}_{1}$ and $\mathcal{A}_{2}$ are whales, then $\Omega_{\mathcal{A}_{1}} \cup \Omega_{\mathcal{A}_{2}} \subset \Omega_{\mathcal{A}_{1} \cap \mathcal{A}_{2}}$. If $\mathcal{A}_{1}$ and $\mathcal{A}_{2}$ are whales and $\mathcal{A}_{1} \subset \mathcal{A}_{2}$, then the Riesz space $\Omega_{\mathcal{A}_{2}}$ is a Riesz ideal in $\Omega_{\mathcal{A}_{1}}$ (as a consequence of (2) in Lemma 3 ). It follows that $\Omega$ can be given the structure of a Riesz space in which every $\Omega_{\mathcal{A}}$ is a Riesz ideal. Then $\Omega$ must be Dedekind complete.

In particular $\Omega_{\mathcal{B}}$ (which is $E_{\mathcal{B}}$ ) is an ideal in $\Omega$. This ideal is order dense. Indeed, let $a \in \Omega, a>0$. Choose a whale $\mathcal{A}$ with $a \in \Omega_{\mathcal{A}}$ and an $A \in \mathcal{A}$ with $a(A)>0$. Then

$$
x:=(a(A \cap B))_{B \in \mathcal{B}}
$$


is an element of $\Omega_{\mathcal{B}}$ and $0<x \leq a$. Thus,

$$
x \mapsto \text { equivalence class of }\left(x_{B}\right)_{B \in \mathcal{B}}
$$

is a Riesz isomorphism of $E$ onto an order-dense ideal of $\Omega$.

So far we have the following result.

Lemma 5. The map

$$
x \mapsto \text { equivalence class of }\left(x_{A}\right)_{A \in \mathcal{B}}
$$

is a Riesz isomorphism of $E$ into an order dense Riesz ideal of the Dedekind complete Riesz space $\Omega$.

It remains to prove that $\Omega$ is laterally complete.

Lemma 6. $\Omega$ is laterally complete.

Proof. Let $\Sigma$ be a disjoint subset of $\Omega^{+}$. For $\sigma \in \Sigma$ we define

$$
\mathcal{A}_{\sigma}=\left\{A \in \mathcal{B}: A \in \operatorname{Dom}_{\bar{\sigma}} \text { and } A \text { is the band generated by } \bar{\sigma}(A)\right\} \text {. }
$$

If $A \in \mathcal{A}_{\sigma}, B \in \mathcal{B}$ and $B \subset A$, then $B$ is the band generated by $\bar{\sigma}(A)$, which is $\bar{\sigma}(B)$; so $B \in \mathcal{A}_{\sigma}$ and $A_{\sigma}$ satisfies condition (1) in the definition of a whale. Consequently, if we put

$$
\mathcal{A}_{0}:=\left\{B \in \mathcal{B}: B \perp A \text { for all } A \in \bigcup_{\sigma \in \Sigma} \mathcal{A}_{\sigma}\right\},
$$

then

$$
\mathcal{A}:=\bigcup_{\sigma \in \Sigma} \mathcal{A}_{\sigma} \cup \mathcal{A}_{0}
$$

is a whale. By the disjointness of $\Sigma$ we can define

$$
\begin{gathered}
x(A)=\bar{\sigma}(A) \quad \text { if } A \in \mathcal{A}_{\sigma} \text { and } \sigma \in \Sigma, \\
x(B)=0 \quad \text { if } B \in \mathcal{A}_{0} .
\end{gathered}
$$

Then $x \in E_{\mathcal{A}}$. The equivalence class $\xi \in \Omega$ to which $x$ belongs is the least upper bound of $\Sigma$.

Lemmas 5 and 6 yield the desired result.

Theorem 7. $\Omega$ is a universal completion of $E$.

\section{SOME CONCLUDING REMARKS}

1. The procedure outlined in this paper works just as well for producing universal completions of Archimedean lattice-ordered groups.

2. Some other papers have produced constructions of universal completions. We mention in particular the interesting approach by de Pagter via extended orthomorphisms in [11], the much quoted paper [5] by Bernau and the outline in [3] going back to Conrad. All of these constructions at one point or another use a maximal disjoint system. The usefulness of maximal disjoint systems is not unlike the convenience of maximal orthonormal subsets in a Hilbert space. The precise relationships of the existence of either of these orthogonal systems to each other and the Axiom of Choice are unclear at this point of time. 
3. If one uses the Axiom of Choice, then a complete disjoint system in $\Omega$ produces a weak order unit. On the other hand, the existence of a weak order unit is easily seen to be equivalent to the existence of a choice function

$$
\mathcal{B} \backslash\{0\} \rightarrow E^{+} \backslash\{0\} .
$$

On its turn the existence of a weak order unit in $\Omega$ can be combined with information from [7] to yield the Maeda-Ogasawara Theorem under the assumption of the Boolean Prime Ideal Theorem. Whether the existence of the above choice function is implied by the Boolean Prime Ideal Theorem is unknown to the authors.

4. It is possible (using the Maeda-Ogasawara Representation Theorem) to put the structure of a semiprime Archimedean $f$-algebra on the universal completion of $E$. Whether the Boolean Prime Ideal Theorem suffices to produce the same effect is another question.

5. What has been constructed in this paper is a universal completion in the sense of [1]. Without assuming the Axiom of Choice our universal completion may not be minimal. Of course one can create a minimal universal completion by taking an appropriate intersection of subspaces.

\section{REFERENCES}

1. Charalambos D. Aliprantis and Owen Burkinshaw, Locally solid Riesz spaces, Academic Press, New York, San Francisco, and London 1978. MR 58:12271

2. __ Positive operators, Academic Press, New York, 1985. MR 87h:47086

3. Marlow Anderson and Todd Feil, Lattice ordered groups, an introduction, Reidel, Dordrecht, 1988. MR 90b:06001

4. Simon J. Bernau, Lateral completion for arbitrary lattice groups, Bull. Amer. Math. Soc. 80 (1974), 334-336. MR 48:10938

5. 263-289. MR 52:5513

6. - Lateral and Dedekind completion of Archimedean lattice groups, J. London Math. Soc. $12(1975 / 76), 320-322$. MR 53:5406

7. G. Buskes and A. van Rooij, Riesz spaces and the ultrafilter theorem. I, Compositio Math. $\mathbf{8 3}$ (1992), 311-327. MR 93g:46006

8. Paul Conrad, The lateral completion of a lattice-ordered group, Proc. London Math. Soc. 3 (1969), 444-480. MR 39:5442

9. D. H. Fremlin, Inextensible Riesz spaces, Math. Proc. Cambridge Philos. Soc. 77 (1975), 71-89. MR 50:7995

10. W. A. J. Luxemburg and A. C. Zaanen, Riesz spaces. I, North-Holland, Amsterdam, 1971. MR 58:23483

11. B. de Pagter, The space of extended orthomorphisms in a Riesz space, Pacific J. Math. 112 (1984) 193-210. MR 85i:46013

12. A. C. Zaanen, Another construction of the universal completion, Indag. Math. 45 (1983), 435-441. MR 85j:46011

Department of Mathematics, University of Mississippi, University, Mississippi 38677

Mathematical Institute, The University of Nijmegen, Toernooiveld, 6525 ED NiJMegen, The Netherlands 